\title{
$\beta$-Catenin Expression Negatively Correlates with WIF1 and Predicts Poor Clinical Outcomes in Patients with Cervical Cancer
}

\author{
Jinxiao Liang, ${ }^{1,2}$ Hui Zhou, ${ }^{1}$ Yongpai Peng, ${ }^{1}$ Xiaofei Xie, ${ }^{1}$ Ruixin Li, ${ }^{1}$ Yunyun Liu, ${ }^{1}$ \\ Qingsheng Xie, ${ }^{1,2}$ and Zhongqiu Lin ${ }^{1}$ \\ ${ }^{1}$ Department of Gynecologic Oncology, Sun Yat-Sen Memorial Hospital, Sun Yat-Sen University, Guangzhou, China \\ ${ }^{2}$ Key Laboratory of Malignant Tumor Gene Regulation and Target Therapy of Guangdong Higher Education Institutes, \\ Sun Yat-Sen University, Guangzhou, China
}

Correspondence should be addressed to Qingsheng Xie; qingshengxie@126.com

and Zhongqiu Lin; linzhqiu@mail.sysu.edu.cn

Received 23 August 2016; Accepted 28 September 2016

Academic Editor: Anne Hamburger

Copyright (c) 2016 Jinxiao Liang et al. This is an open access article distributed under the Creative Commons Attribution License, which permits unrestricted use, distribution, and reproduction in any medium, provided the original work is properly cited.

\begin{abstract}
Aberrant activation of the canonical Wnt pathway plays a significant role in cervical cancer (CC). However, limited data show the correlation between the cancer clinicopathological characteristics and the key molecules such as $\beta$-catenin and Wnt inhibitory factor 1 (WIF1). In this study, $\beta$-catenin and WIF1 expression were analyzed by immunohistochemistry for 196 patients with CC, 39 with cervical intraepithelial neoplasia (CIN), and 41 with normal cervical epithelium (NCE). Significant overexpression of $\beta$-catenin was detected in CC (67.9\%) when compared to CIN (43.6\%) or NCE (34.1\%), $p<0.01$, while low WIF1 expression was detected in CC $(24.0 \%)$ when compared to CIN $(59.0 \%)$ or NCE $(58.5 \%), p<0.001$. Negative correlation was shown between $\beta$-catenin and WIF1 expression $(r=-0.637, p<0.001)$. In addition, multivariate analysis revealed that both lymph node metastasis and $\beta$ catenin expression were the independent prognostic factors not only for disease-free survival $(\mathrm{HR}=5.029, p<0.001 ; \mathrm{HR}=2.588$, $p=0.035$, resp.), but also for overall survival ( $\mathrm{HR}=5.058, p<0.001 ; \mathrm{HR}=2.873, p=0.031$, resp.). Our findings indicate that, besides lymph node metastasis, $\beta$-catenin expression may also be a poor prognostic factor for CC while WIF1 could be a potential drug target for treatment of advanced CC.
\end{abstract}

\section{Introduction}

Cervical cancer (CC) is the fourth most common cancer in women. According to existing data, there were 528,000 registered new cases and 266,000 deaths only in year 2012 [1]. Most patients with early CC have good prognosis. By contrast, patients with a later cancer stage or metastatic CC have poor survival rate because of less effective treatments available [2]. Therefore, additional studies on late cancer development and prognosis methods are necessary.

Aberrant activation of the Wingless-type (Wnt)/ $\beta$-catenin (canonical Wnt pathway) is a very common pathway in human CC [3]. Recent molecular testing has demonstrated that the CC biological behavior may arise as a multistep gene process. Specifically, infection with human papillomavirus (HPV) could be "the first hit" [4], while the dysregulation of canonical Wnt pathway may be required as "the second hit" in cervical oncogenesis $[3,5,6]$. However, the mechanism involving Wnt pathway in CC is still not well understood and requires additional studies. In the canonical Wnt pathway, the binding of Wnts to a heterodimeric receptor complex stabilizes the $\beta$-catenin expression and leads to the activation of $\beta$-catenin target genes inside the nucleus $[7,8]$. Furthermore, Wnt inhibitory factor 1 (WIF1) is an upstream secreted Wnt antagonist, first identified as highly conserved gene in the human retina [9]. WIF1 main function is to bind the extracellular Wnt ligands [10], disturbing the Wnt interaction with the receptors [7] and 
TABLE 1: Comparison of WIF1 and $\beta$-catenin expression in NCE, CIN, and CC.

\begin{tabular}{lcccccc}
\hline Variable & Cases (number) & & $\beta$-Catenin & & \multicolumn{2}{c}{ WIF1 } \\
& & Negative & Positive & $p^{\mathrm{a}}$ & Negative & $p^{\mathrm{a}}$ \\
\hline CC & 196 & 63 & $133(67.9 \%)$ & & 149 & $47(24.0 \%)$ \\
CIN & 39 & 22 & $17(43.6 \%)$ & $<\mathbf{0 . 0 1}^{\mathrm{b}}$ & 16 & $23(59.0 \%)$ \\
NCE & 41 & 27 & $14(34.1 \%)$ & & 17 & $24(58.5 \%)$ \\
\hline
\end{tabular}

${ }^{\mathrm{a}}$ The $p$ value was determined using the $\chi^{2}$ test. ${ }^{\mathrm{b}} p<0.01$ was found in the ratio of $\beta$-catenin positive expression when compared to CC and CIN or CC and NCE; ${ }^{c} p<0.001$ was found in the ratio of WIFl positive expression when compared to CC and CIN or CC and NCE; no significant differences were observed when comparing CIN and NCE.

consequently leading to $\beta$-catenin degradation, therefore inhibiting the canonical Wnt pathway. Currently, there are dozens of studies regarding the correlation with $\beta$-catenin and various types of cancer $[7,11]$. In most of these cases, elevated levels of $\beta$-catenin have been strongly correlated with poor cancer prognosis. However, so far, there have been limited reports focusing on the association between $\beta$-catenin, WIF1, and the clinicopathological characteristics in CC.

In the present study, we investigated the association between the canonical Wnt pathway $(\beta$-catenin as the hallmark [7]), WIF1, and clinicopathological features of 196 patients with CC and analyzed their prognostic value in CC.

\section{Materials and Methods}

2.1. Patients and Tissue Samples. Between December 2002 and October 2007, 196 patients from the Department of Gynecologic Oncology (Sun Yat-Sen Memorial Hospital, Canton, China), diagnosed with CC (stages IA-IIB) and submitted to curative surgical resection, were recruited for this study. Cases were restricted to those who did not receive any tumor related treatment before surgery. The clinicopathological characteristics were summarized in Table 3. Furthermore, an additional 39 cervical intraepithelial neoplasia (CIN) and 41 normal cervical epithelium (NCE) samples were obtained from women undergoing hysterectomy for noncancerous diagnosis. Histological diagnosis and tumor stage and grade were determined according to the World Health Organization (WHO) and the International Federation of Gynecology and Obstetrics (FIGO) staging systems [12,13]. All specimens were anonymously coded in accordance with local ethical guidelines (as stipulated by the Declaration of Helsinki). All study protocols were approved by the University Ethics Committee.

2.2. Immunohistochemistry (IHC) and Samples Evaluation. According to the previously described method [14, 15], IHC analysis was performed using anti- $\beta$-catenin antibody (CST, \#9562, Boston, MA, America) and anti-WIF1 antibodyN-terminal (Abcam, ab71204, Cambridge, MA, America). Human breast tissues were used as positive controls; negative controls were obtained by replacing the primary antibodies with phosphate buffered saline. The staining of $\beta$-catenin was evaluated according to Maruyama's method [16]. If more than $10 \%$ of cancer cells were positively stained for cytoplasm and/or nuclei, the cells were regarded as $\beta$-cateninpositive expression. By contrast, membrane staining was only classified as $\beta$-catenin-negative expression; for WIF1 protein expression, nuclear staining was considered to be negative [17]. Finally, if more than $10 \%$ of cancer cells were positively stained for WIF1 in cytoplasmic and/or on cell membranes, the positive IHC results were recorded.

2.3. Statistical Analysis. All the statistical analysis was conducted using SPSS software, version 13.0 (SPSS Inc., Chicago, IL, USA). Associations between the clinicopathological characteristics and the pattern of WIF1 and $\beta$-catenin expression were examined using Pearson's $\chi^{2}$ test. Survival rates were calculated using the Kaplan-Meier method and compared using the log-rank test. Univariate and multivariate survival analyses were performed using the Cox regression model for DFS and OS. A forward stepwise procedure was used to identify independent variables in the multivariate analysis. $p<0.05$ indicated statistical significance.

\section{Results}

3.1. IHC of $\beta$-Catenin and WIF1 and Their Correlation with CC. As shown in Figure 1 and Table 1, obvious membranous staining of $\beta$-catenin and nuclear staining of WIF1 were observed in most cancer and noncancerous samples; however, both staining patterns were considered to be negatively expressed. No significant difference was observed between $\beta$-catenin-positive expression ratios (cytoplasmic/nuclear staining) in CIN $(17 / 39,43.6 \%)$ compared to NCE (14/41, $34.1 \%)$. In addition, positive $\beta$-catenin expression was found in $(133 / 196,67.9 \%)$ cervical cancer samples (both with $p<$ 0.01). Furthermore, low expression of WIF1 was significant in CC samples compared with CIN and NCE $(p<0.001)$. Briefly, from 149 WIF1 negative CC samples, 126 (84.6\%) were $\beta$-catenin-positive. On the other hand, from 47 WIF1 positive CC samples, $40(85.1 \%)$ were $\beta$-catenin-negative $(p<0.001)$, Table 2 . Statistically, $\beta$-catenin expression had negative correlation compared to WIF1 expression $(r=$ $-0.637 ; p<0.001)$.

3.2. IHC of $\beta$-Catenin and WIF1 in Representative Cases with CC. Images of WIF1 and $\beta$-catenin staining for representative CC cases were shown in Figure 2, which indicated that, with the decreased expression of WIF1, clearly increased expression of $\beta$-catenin was observed. Specifically, 


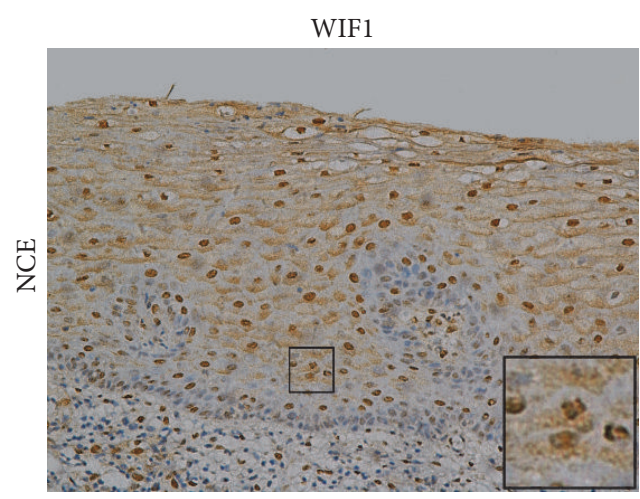

(a)

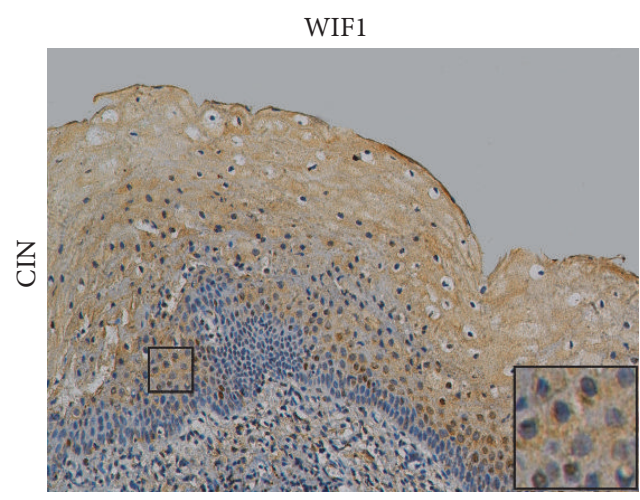

(c)

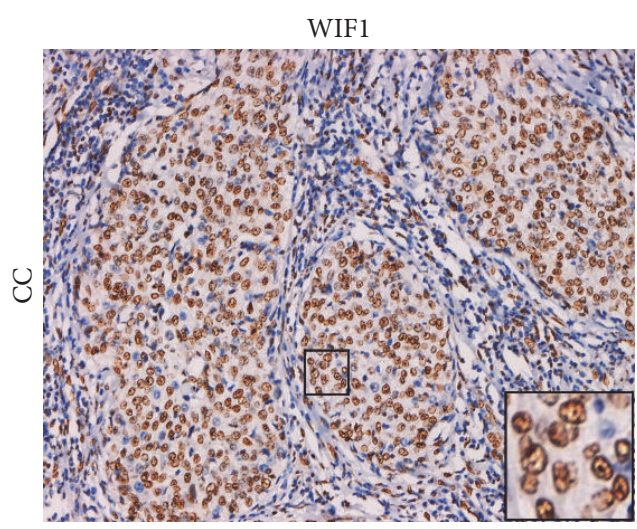

(e)

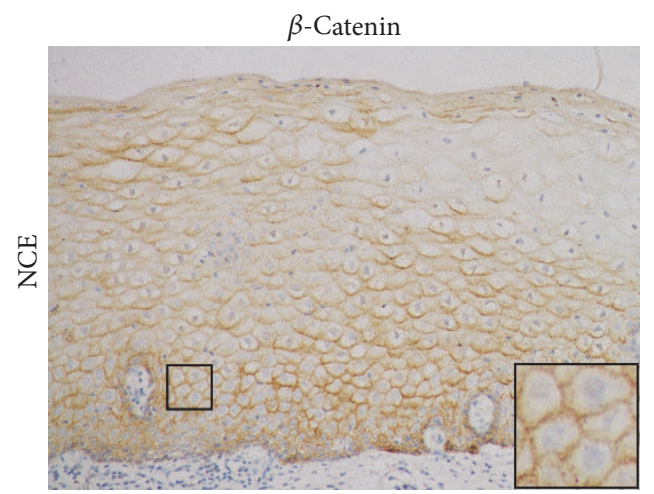

(b)

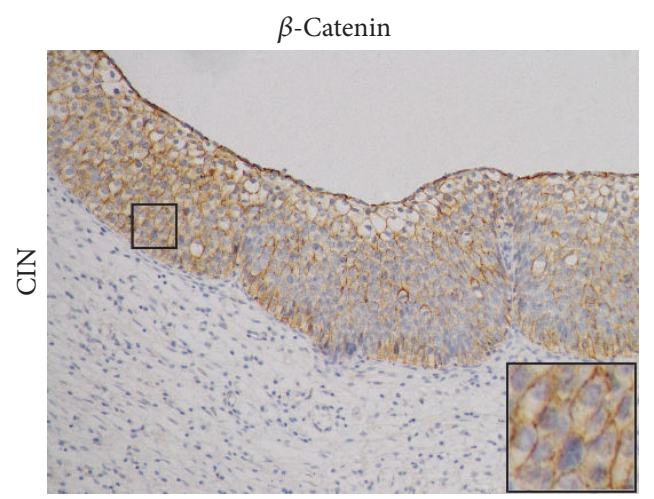

(d)

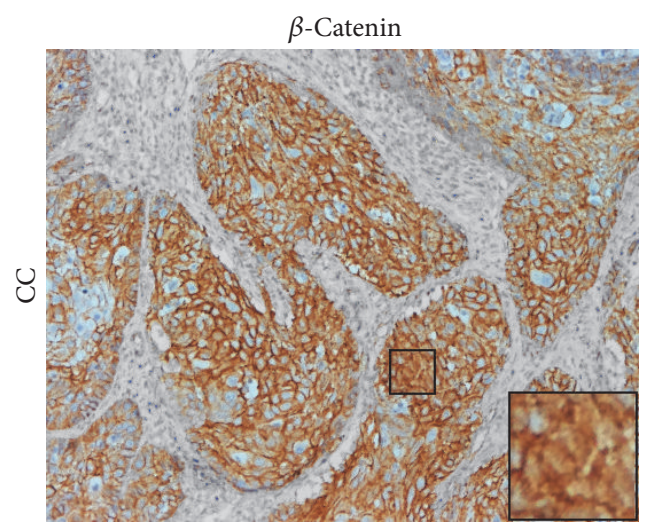

(f)

FIGURE 1: WIF1 and $\beta$-catenin staining images of human cervical tissues by IHC. ((a), (c)) Positive cytoplasmic staining of WIF1 was observed in both NCE and CIN. ((b), (d)) Positive membranous staining with no cytoplasmic/nuclear staining of $\beta$-catenin was observed in both NCE and CIN. (e) Positive nuclear staining without membranous/cytoplasmic staining of WIF1 was observed in CC. (f) Positive cytoplasmic staining of $\beta$-catenin was observed in CC. Magnification: $\times 200$ (hematoxylin counterstained). Insets are magnified images from selected areas (small squares).

the expression of $\beta$-catenin was usually absent in patients who had the most intense cytoplasmic WIF1 staining while the staining of $\beta$-catenin was intense in those who had no cytoplasmic expression of WIF1.

3.3. Special WIF1 Staining Pattern of CC Cells with Mitotic Figures. WIF1 was usually found to be moderate-to-strong nuclear staining but without cytoplasmic staining in this study. However, as shown in Figure 3, another pattern of WIF1 staining was observed in mitotic figures in some CC samples, which were characterized by blue-staining (hematoxylin stain) nuclei and brown-staining (anti-WIF1 stain) cytoplasm and were registered for the first time. The individual mitotic figures could present pleomorphic appearances, such as 


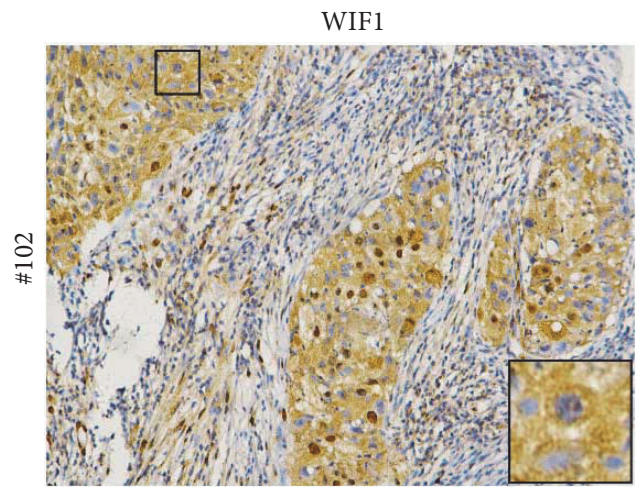

(a)

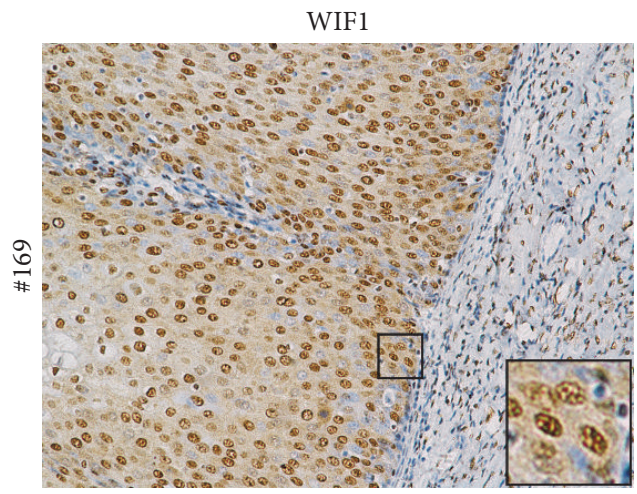

(c)

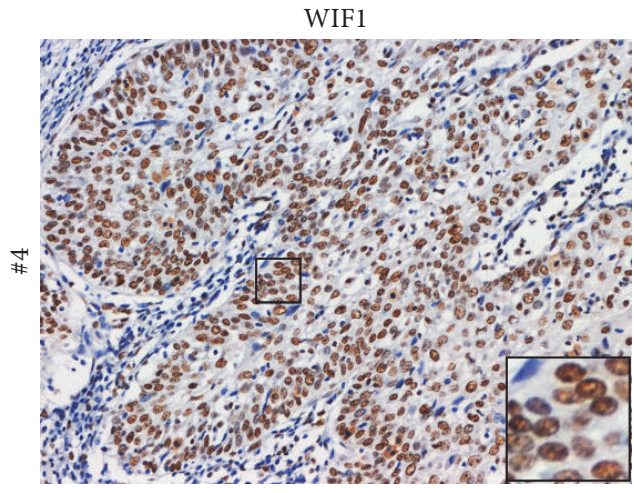

(e)

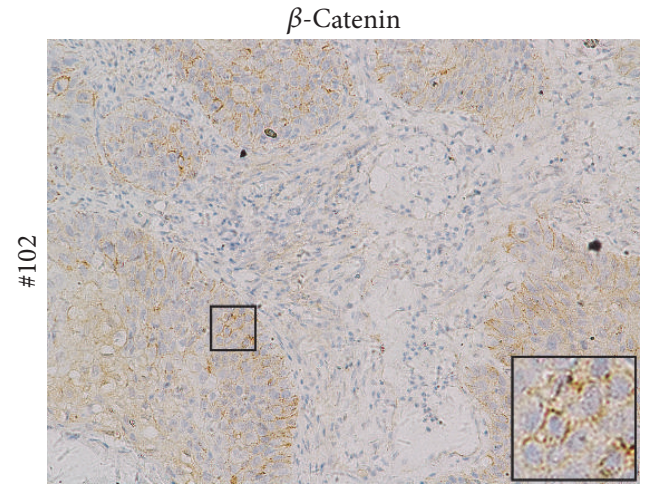

(b)

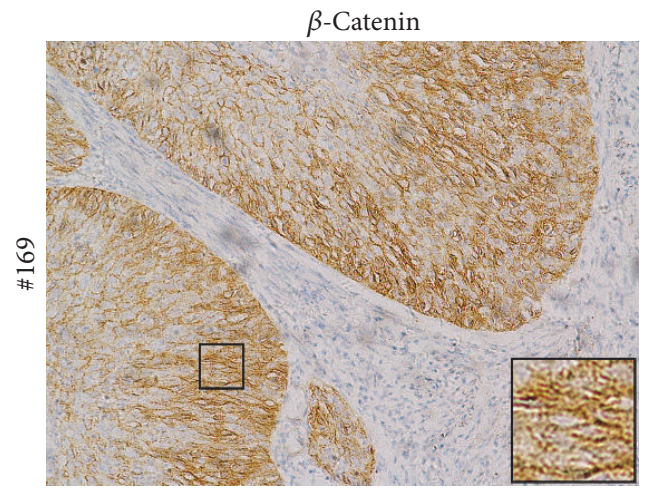

(d)

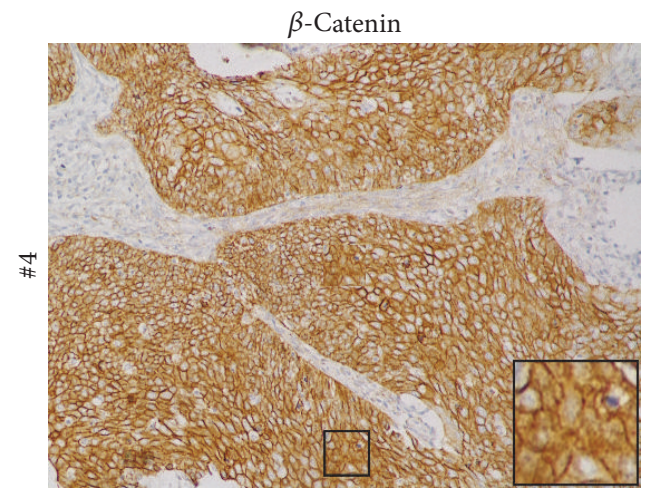

(f)

FIgURE 2: Comparison of WIF1 and $\beta$-catenin expression in CC. The relationship of WIF1 and $\beta$-catenin immunostaining for three representative cases: ((a), (b)) Case 102, indicating strong WIF1 cytoplasmic staining and negative $\beta$-catenin cytoplasmic/nuclear staining; ((c), (d)) Case 169, which shows moderate immunostaining for both WIF1 and $\beta$-catenin; ((e), (f)) Case 4, showing negative WIF1 cytoplasmic staining and strong $\beta$-catenin cytoplasmic staining. Magnification: $\times 200$. Insets are magnified images from selected areas (small squares).

TABLE 2: The relationship of WIF1 and $\beta$-catenin in CC.

\begin{tabular}{ccccc}
\hline \multirow{2}{*}{ Variable } & \multicolumn{2}{c}{ WIF1 } & $p^{\mathrm{a}}$ & Correlation coefficient \\
& $(-)$ & $(+)$ & & \\
\hline$\beta$-Catenin & & & & -0.637 \\
$(-)$ & 23 & 40 & $<\mathbf{0 . 0 0 1}$ & \\
$(+)$ & 126 & 7 & & \\
\hline
\end{tabular}

${ }^{\mathrm{a}}$ The $p$ value was determined using the $\chi^{2}$ test. mirror-image cells (Figure 3(a)) and sunflower-like cells (Figure 3(b)).

3.4. The Expression of WIF1 and $\beta$-Catenin and Their Association with the Clinicopathological Features of CC. As shown in Table 3, $\beta$-catenin-positive expression was associated with a higher rate of lymphovascular space invasion $(p=0.017)$. Furthermore, WIF1 positive staining was associated with less 
TABLE 3: Association between the expression of WIF1 and $\beta$-catenin and clinicopathological parameters of CC (stages IA-IIB).

\begin{tabular}{|c|c|c|c|c|c|c|c|c|}
\hline \multirow{2}{*}{ Variable } & \multicolumn{2}{|c|}{ Cases } & \multicolumn{3}{|c|}{ WIF1 } & \multicolumn{3}{|c|}{$\beta$-Catenin } \\
\hline & Number & $(\%)$ & Negative & Positive & $p^{\mathrm{a}}$ & Negative & Positive & $p^{\mathrm{a}}$ \\
\hline \multicolumn{9}{|l|}{ Histologic subtype } \\
\hline Squamous cell carcinoma & 172 & 87.8 & 133 & 39 & \multirow{2}{*}{ NS } & 58 & 114 & \multirow{2}{*}{ NS } \\
\hline Adenocarcinoma & 24 & 12.2 & 16 & 8 & & 5 & 19 & \\
\hline \multicolumn{9}{|l|}{ FIGO stage } \\
\hline IA & 16 & 8.2 & 13 & 3 & \multirow{4}{*}{ NS } & 6 & 10 & \multirow{4}{*}{ NS } \\
\hline IB1, IIA1 & 102 & 52.0 & 77 & 25 & & 31 & 71 & \\
\hline IB2, IIA2 & 64 & 32.7 & 46 & 18 & & 25 & 39 & \\
\hline IIB & 14 & 7.1 & 13 & 1 & & 1 & 13 & \\
\hline \multicolumn{9}{|l|}{ Tumor grade* } \\
\hline G1 & 35 & 19.2 & 27 & 8 & \multirow{2}{*}{ NS } & 6 & 29 & \multirow{2}{*}{ NS } \\
\hline G2/G3 & 147 & 80.8 & 112 & 35 & & 41 & 106 & \\
\hline \multicolumn{9}{|l|}{ Parametrial invasion } \\
\hline Negative & 186 & 94.9 & 141 & 45 & \multirow{2}{*}{ NS } & 62 & 124 & \multirow{2}{*}{ NS } \\
\hline Positive & 10 & 5.1 & 8 & 2 & & 1 & 9 & \\
\hline \multicolumn{9}{|l|}{ Surgical margin involved } \\
\hline No & 189 & 96.4 & 143 & 46 & \multirow{2}{*}{ NS } & 63 & 126 & \multirow{2}{*}{ NS } \\
\hline Yes & 7 & 3.6 & 6 & 1 & & 0 & 7 & \\
\hline \multicolumn{9}{|l|}{ Lymph node metastasis } \\
\hline Negative & 142 & 72.4 & 103 & 39 & \multirow{2}{*}{ NS } & 51 & 91 & \multirow{2}{*}{ NS } \\
\hline Positive & 54 & 27.6 & 46 & 8 & & 12 & 42 & \\
\hline \multicolumn{9}{|l|}{ Tumor size $(\mathrm{cm})$} \\
\hline$\leq 4$ & 117 & 59.7 & 90 & 27 & \multirow{2}{*}{ NS } & 36 & 81 & \multirow{2}{*}{ NS } \\
\hline$>4$ & 79 & 40.3 & 59 & 20 & & 27 & 52 & \\
\hline \multicolumn{9}{|l|}{ Cervical stromal invasion } \\
\hline$\leq$ one second & 86 & 43.9 & 56 & 30 & \multirow{2}{*}{0.002} & 27 & 59 & \multirow{2}{*}{ NS } \\
\hline$>$ one second & 110 & 56.1 & 93 & 17 & & 34 & 76 & \\
\hline Lymphovascular invasion* & & & & & & & & \\
\hline Negative & 93 & 61.6 & 64 & 29 & 0.035 & 33 & 60 & 0.017 \\
\hline Positive & 58 & 38.4 & 49 & 9 & 0.00 & 10 & 48 & \\
\hline Age (years) & & & & & & & & \\
\hline$\leq 35^{* *}$ & 35 & 17.9 & 30 & 5 & NS & 9 & 26 & NS \\
\hline$>35$ & 161 & 82.1 & 119 & 42 & 100 & 54 & 107 & \\
\hline
\end{tabular}

NS: not significant; HPF: high-power field. ${ }^{a}$ The $p$ value was determined using the $\chi^{2}$ test. Significant $p$ values are shown with bold font. ${ }^{*}$ The number of patients is less than 196 because of missing data. ${ }^{* *}$ Age (range: $23-66$ y, median: $42 \mathrm{y}$ ).

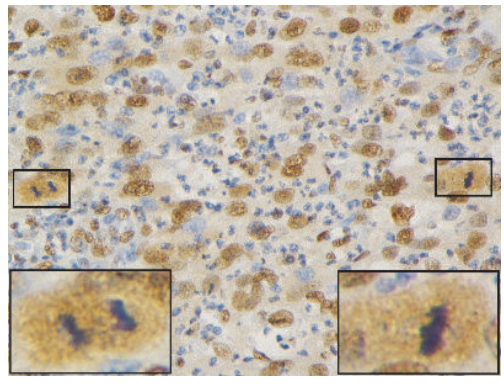

(a)

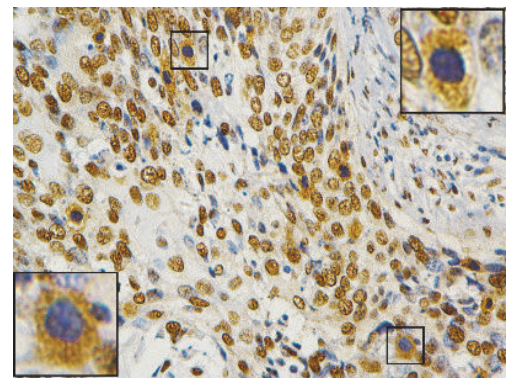

(b)

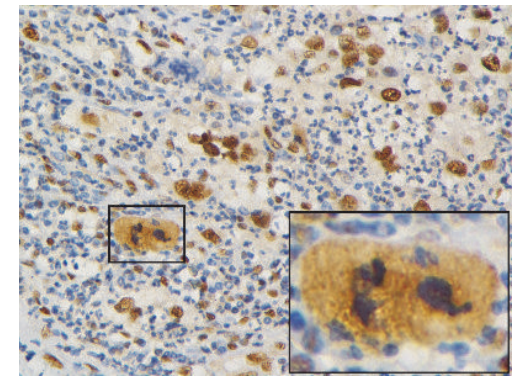

(c)

FIGURE 3: Pleomorphic WIF1 staining appearances of CC cells with mitotic figures. The blue-stained (hematoxylin stain) nuclei and brownstained (WIF1 stain) cytoplasm were characteristic. (a) CC cells in metaphase with small short rod-like nuclei and medium-sized cytoplasm were shown on the right while CC cells in telophase with mirror image were seen on the left. (b) CC cells in prophase with round nuclei and intermediate-sized cytoplasm (sunflower-like appearance) were shown. (c) CC cells in triploid mitotic figures with apparently lobulated nuclei and abundant brown-stained cytoplasm were shown. Magnification: $\times 400$. Insets are magnified images from selected areas (small squares). 


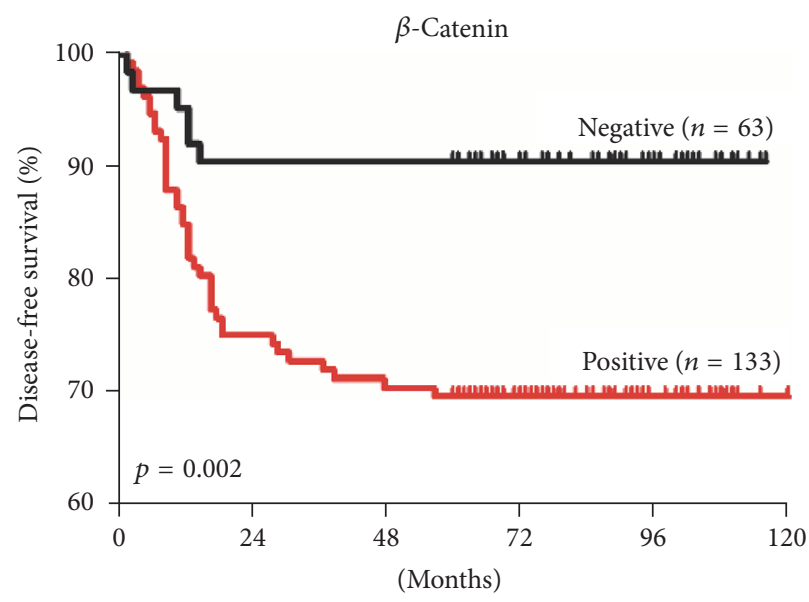

(a)

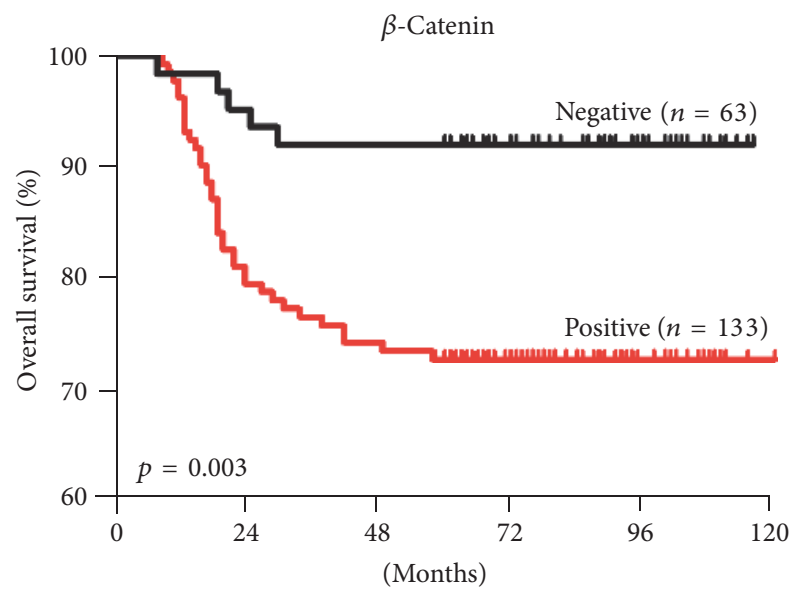

(c)

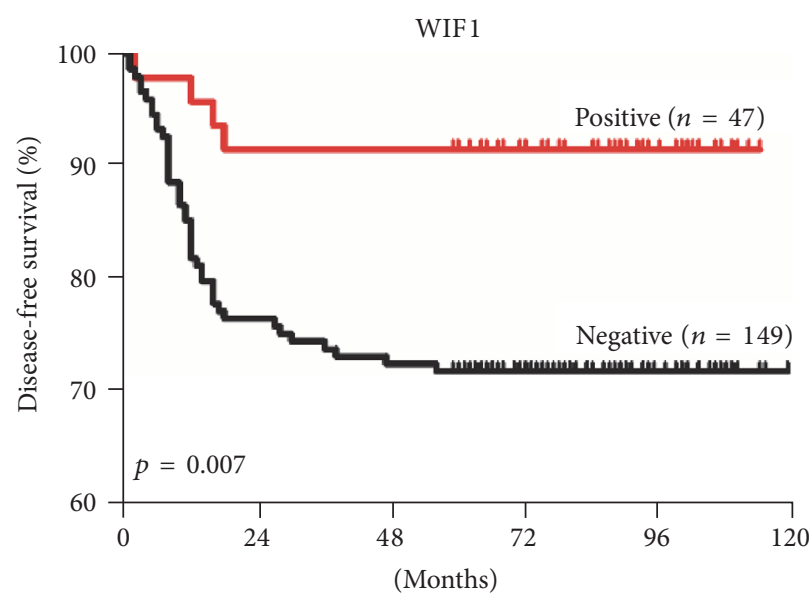

(b)

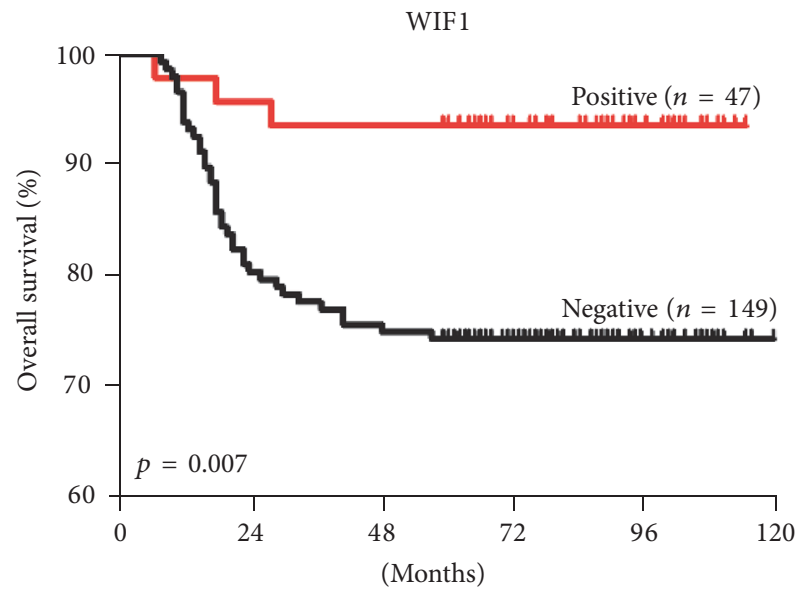

(d)

Figure 4: Kaplan-Meier 5-year disease-free survival (DFS) and 5-year overall survival (OS) curves for patients with cervical cancer. ((a) and (c)) Patients with $\beta$-catenin-positive tumors tended to have poorer DFS and OS. ((b) and (d)) Patients with WIF1-positive expression had significantly better DFS and OS than those with negative results.

cervical stromal invasion $(p=0.002)$ and a lower rate of lymphovascular space invasion $(p=0.035)$.

\subsection{The Clinicopathological Features and Their Prognostic} Values. During the median follow-up of 70 months (range: 60-121 months), 46/196 (23.5\%) patients underwent CC recurrence. Among those patients, 41 (88.1\%) died from cancer progression and 5 patients with recurrent vaginal or lymph node CC survived after surgery and adjuvant concurrent chemoradiotherapy. The 5-year disease-free survival (DFS) and overall survival (OS) rates were $76.5 \%$ and $79.1 \%$, respectively. CC patients with positive $\beta$ catenin expression had poorer 5-year DFS (69.9\% versus $90.5 \%, p=0.002$; Figure $4(\mathrm{a}))$ and 5-year OS $(72.9 \%$ versus $92.1 \%, p=0.003$; Figure $4(\mathrm{c}))$ than patients with negative $\beta$-catenin expression. Patients with positive WIF1 expression had longer 5-year DFS (91.5\% versus 71.8\%, $p=0.007$; Figure $4(\mathrm{~b}))$ and OS $(93.6 \%$ versus $74.5 \%$, $p=0.007$; Figure 4(d)) than those with negative WIF1 results.

The univariate analysis showed that advanced FIGO stage, parametrial invasion, positive surgical margin, lymph node metastasis, larger tumor size $(>4 \mathrm{~cm})$, and $\beta$-catenin expression were correlated with poorer 5-year DFS rate. In addition, lymph node metastasis $(p<0.001$; hazard ratio $(\mathrm{HR})=5.029$; 95\% CI: 2.623-9.645) and $\beta$-catenin expression $(p=0.035$; $\mathrm{HR}=2.588$; 95\% CI: 1.071-6.251) emerged as independent predictors of 5-year DFS in multivariate analysis, Table 4. For 5-year OS, FIGO stage, parametrial invasion, positive surgical margin, lymph node metastasis, larger tumor size, and $\beta$-catenin expression were included in the multivariate analysis. Lymph node metastasis $(p<0.001$; $\mathrm{HR}=5.058$; 95\% CI: $2.524-10.137)$ and $\beta$-catenin expression $(p=0.031$; $\mathrm{HR}=2.873$; $95 \%$ CI: $1.102-7.492)$ emerged as independent predictors of 5-year OS, Table 4. 
TABLE 4: Univariate and multivariate analysis of factors associated with DFS and OS for patients with CC.

\begin{tabular}{|c|c|c|c|c|c|c|c|c|}
\hline \multirow{3}{*}{ Variable } & \multicolumn{4}{|c|}{ 5-year DFS ${ }^{\mathrm{c}}$} & \multicolumn{4}{|c|}{ 5-year OS } \\
\hline & \multirow{2}{*}{$\begin{array}{c}\text { Univariate } \\
\qquad p^{\mathrm{a}}\end{array}$} & \multicolumn{3}{|c|}{ Multivariate $^{\mathrm{d}}$} & \multirow{2}{*}{$\begin{array}{c}\text { Univariate } \\
\qquad p^{\mathrm{a}}\end{array}$} & \multicolumn{3}{|c|}{ Multivariate $^{\mathrm{d}}$} \\
\hline & & $p^{\mathrm{a}}$ & $\mathrm{HR}^{\mathrm{b}}$ & $95 \% \mathrm{CI}$ & & $p^{\mathrm{a}}$ & $\mathrm{HR}^{\mathrm{b}}$ & $95 \% \mathrm{CI}$ \\
\hline \multicolumn{9}{|l|}{ FIGO stage } \\
\hline \multicolumn{9}{|l|}{ IA } \\
\hline IB1, IIA1 & \multirow{3}{*}{$<0.001$} & \multirow{3}{*}{ NS } & & & \multirow{3}{*}{0.002} & \multirow{3}{*}{ NS } & & \\
\hline IB2, IIA2 & & & & & & & & \\
\hline IIB & & & & & & & & \\
\hline \multicolumn{9}{|c|}{ Parametrial invasion } \\
\hline Negative & \multirow[t]{2}{*}{0.002} & \multirow[t]{2}{*}{ NS } & & & \multirow{2}{*}{0.007} & \multirow[t]{2}{*}{ NS } & & \\
\hline Positive & & & & & & & & \\
\hline \multicolumn{9}{|c|}{ Surgical margin involved } \\
\hline No & \multirow{2}{*}{0.008} & \multirow{2}{*}{ NS } & & & \multirow{2}{*}{0.036} & \multirow{2}{*}{ NS } & & \\
\hline Yes & & & & & & & & \\
\hline \multicolumn{9}{|c|}{ Lymph node metastasis } \\
\hline Negative & \multirow[t]{2}{*}{$<0.001$} & \multirow[t]{2}{*}{$<0.001$} & \multirow[t]{2}{*}{5.029} & \multirow{2}{*}{$2.623-9.645$} & \multirow[t]{2}{*}{$<0.001$} & \multirow{2}{*}{$<0.001$} & \multirow{2}{*}{5.058} & \multirow{2}{*}{$2.524-10.137$} \\
\hline Positive & & & & & & & & \\
\hline \multicolumn{9}{|c|}{ Tumor size $(\mathrm{cm})$} \\
\hline$\leq 4$ & $<0.001$ & NS & & & 0.001 & NS & & \\
\hline$>4$ & -0.001 & No & & & & & & \\
\hline$\beta$-Catenin & & & & & & & & \\
\hline Negative & 0.001 & 0.035 & 2.588 & $1.071-6.251$ & 0.002 & 0.031 & 2.873 & $1.102-7.492$ \\
\hline Positive & & & & & & & & \\
\hline
\end{tabular}

DFS: disease-free survival; OS: overall survival; HR: hazard ratio; CI: confidence interval; NS: not significant; HPF: high-power field. ${ }^{a}$ Significant $p$ values are shown in bold font. ${ }^{b} \mathrm{HR}>1$ indicates that risk for recurrence/death increased; HR $<1$ indicates that risk for recurrence/death decreased. ${ }^{c}$ Univariate and multivariate analyses and Cox proportional hazards regression model. ${ }^{\mathrm{d}}$ Variables associated with survival by univariate analysis were adopted as covariates in multivariate analyses.

\section{Discussion}

Dysregulation of Wnt pathway is involved in different diseases, including cancer. $\beta$-Catenin, the key factor of canonical Wnt pathway, conducts Wnt signals to the nucleus and upregulates oncogenes during tumorigenesis $[7,8]$. It has been demonstrated that the expression of $\beta$-catenin can be upregulated in various cancers, including CC $[3,5,18]$. Consistent with previous reports [19-21], $\beta$-catenin accumulation inside the cytoplasm was found to be significantly increased in CC when compared with CIN and NCE in this study. In addition, by Cox regression analysis, it was shown that $\beta$ catenin-positive expression was significantly correlated with poor prognosis in 5-year DFS and 5-year OS. In the present study, a negative correlation between cytoplasmic/nuclear $\beta$ catenin accumulation and cytoplasmic WIF1 immunostaining (positive expression) was found. In addition, decreased WIF1 expression in CC was consistent with some previous studies which were based on human tumor study, such as gastrointestinal tract, kidney, glioblastoma, osteosarcoma, lung, pituitary, bladder, and oral cavity [17, 22-28]. Therefore, we hypothesize that the canonical Wnt pathway was activated, whereas the Wnt antagonist WIF1 was inhibited, by the multistep gene process in CC.

Our results have suggested that WIF1 expression was negative in $94.7 \%$ of CC samples with $\beta$-catenin-positive expression (Table 2), while the results in Figure 2 support the association between downregulation of WIF1 and upregulation of $\beta$-catenin expression in CC. This may indicate that inactivation of WIF1 and accumulation of stabilized $\beta$-catenin are a gradual process during tumorigenesis and progression of CC. Our study showed that the positive staining of WIF1 was significantly reduced in patients with $>1 / 2$ cervical stromal invasion and lymphovascular space invasion, while positive staining of $\beta$-catenin was associated with lymphovascular space invasion. Furthermore, though no statistical significance was registered, the expression of WIF1 was much lower in the patients with surgical margin involvement (14.3\%) and lymph node metastasis (14.8\%) than in those with no surgical margin involvement $(24.3 \%)$ or no lymph node metastasis (27.5\%; Table 3 ). These results stand in support of the idea that WIF1 downregulation is an early event [6] and can potentially inhibit the early progression stage of CC by antagonizing canonical Wnt pathway.

Recurrence in many advanced cancers has been associated with chemoresistance. The related mechanisms include tumor angiogenesis, maintenance of resistant cancer stem cells, dysregulation of cell cycle, and defects in apoptosis, which are all at least partly regulated by the canonical Wnt pathway [29]. The expressions of components related to this pathway are frequently altered. Increased expression of Wnt ligands was reported in breast cancer [30] and dishevelled in 
cervical cancer [31], while decreased expression of dickkopf1 was reported in pancreatic cancer [32], secreted frizzledrelated protein 1 (SFRP1) in lung cancer [33], and WIF1 in cervical cancer [6]. The potential therapeutic targets related to these compounds in cancer have been investigated through successful preclinical studies [34]. Therefore, just as in some other carcinomas [22, 35-37], WIF1 is a potent drug target in CC treatment.

Although the cytoplasmic WIF1 staining pattern was consistent with previous studies $[6,14,15]$, the moderateto-strong brown nuclear staining of WIF1 was common in our study. In some CC cases, mitotic figures were cytoplasmpositive and nuclei-negative staining (Figure 3 ), which made them easily identifiable compared to nonmitotic cancer cells. These results indicate that the changes of the localization of WIF1 expression may be related to the uncontrolled CC cell division. However, at present, the reason of opposite cytoplasmic/nuclear WIF1 staining patterns between mitotic figures and nonmitotic CC cells is unclear. Lack of study on the mechanisms and functions of the translocation of WIF1 between mitotic figures and nonmitotic CC cells is a limitation of our study. Further investigations for the reason are needed.

\section{Conclusions}

Our results have demonstrated the upregulation of $\beta$-catenin and downregulation of WIF1 in CC samples compared to CIN and NCE. Along with clinicopathological characteristics, such as lymph node metastasis and cervical stromal invasion, increased $\beta$-catenin expression has been shown to be a poor prognostic factor for $\mathrm{CC}$, indicating the aberrant activation of canonical Wnt pathway. Moreover, the WIF1 staining pattern in mitotic figures (IHC, cytoplasm-positive and nucleus-negative) was opposite to most other CC cells in this study, and the reason needs to be explored in the future.

\section{Ethical Approval}

This study was approved by the Ethics Committee of Sun YatSen Memorial Hospital (Permit no. 2014-63) and all aspects of the study comply with the Declaration of Helsinki.

\section{Consent}

Each patient (or her next of kin in case the patient is dead) who was enrolled in this study has signed informed consent to participate in this research.

\section{Competing Interests}

The authors declare that there are no competing interests regarding the publication of this paper.

\section{Acknowledgments}

The authors thank all patients who participated in this study. This work was supported by grants from the National Natural Science Foundation of China (30672221, 30872743).

\section{References}

[1] GLOBOCAN 2012 (IARC) Section of Cancer Surveillance, Cervical Cancer: Estimated Incidence, Mortality and Prevalence Worldwide in 2012, World Health Organization, 2016.

[2] D. S. Dizon, H. J. Mackay, G. M. Thomas et al., "State of the science in cervical cancer: where we are today and where we need to go," Cancer, vol. 120, no. 15, pp. 2282-2288, 2014.

[3] A. Üren, S. Fallen, H. Yuan et al., "Activation of the canonical Wnt pathway during genital keratinocyte transformation: a model for cervical cancer progression," Cancer Research, vol. 65, no. 14, pp. 6199-6206, 2005.

[4] C. B. J. Woodman, S. I. Collins, and L. S. Young, "The natural history of cervical HPV infection: unresolved issues," Nature Reviews Cancer, vol. 7, no. 1, pp. 11-22, 2007.

[5] C. Perez-Plasencia, A. Duenas-Gonzalez, and B. AlatorreTavera, "Second hit in cervical carcinogenesis process: involvement of wnt/beta catenin pathway," International Archives of Medicine, vol. 1, no. 1, p. 10, 2008.

[6] I. Ramachandran, E. Thavathiru, S. Ramalingam et al., "Wnt inhibitory factor 1 induces apoptosis and inhibits cervical cancer growth, invasion and angiogenesis in vivo," Oncogene, vol. 31, no. 22, pp. 2725-2737, 2012.

[7] H. Clevers and R. Nusse, "Wnt/ $\beta$-catenin signaling and disease," Cell, vol. 149, no. 6, pp. 1192-1205, 2012.

[8] A. Kikuchi and H. Yamamoto, "Tumor formation due to abnormalities in the $\beta$-catenin-independent pathway of Wnt signaling," Cancer Science, vol. 99, no. 2, pp. 202-208, 2008.

[9] J. C. Hsieh, L. Kodjabachian, M. L. Rebbert et al., "A new secreted protein that binds to Wnt proteins and inhibits their activities," Nature, vol. 398, no. 6726, pp. 431-436, 1999.

[10] Y. Kawano and R. Kypta, "Secreted antagonists of the Wnt signalling pathway," Journal of Cell Science, vol. 116, no. 13, pp. 2627-2634, 2003.

[11] J. N. Anastas and R. T. Moon, "WNT signalling pathways as therapeutic targets in cancer," Nature Reviews Cancer, vol. 13, no. 1, pp. 11-26, 2013.

[12] FIGO Committtee On Gynecologic Oncology, "FIGO staging for carcinoma of the vulva, cervix, and corpus uteri," International Journal of Gynecology \& Obstetrics, vol. 125, no. 2, pp. 9798, 2014.

[13] B. Perunovic, "WHO classification of cervical tumors," 2013.

[14] A. L. Delmas, B. M. Riggs, C. E. Pardo et al., "Wif1 is a frequent target for epigenetic silencing in squamous cell carcinoma of the cervix," Carcinogenesis, vol. 32, no. 11, pp. 1625-1633, 2011.

[15] C. Wissman, P. J. Wild, S. Kaiser et al., "WIF1, a component of the Wnt pathway, is down-regulated in prostate, breast, lung, and bladder cancer," Journal of Pathology, vol. 201, no. 2, pp. 204-212, 2003.

[16] K. Maruyama, A. Ochiai, S. Akimoto et al., "Cytoplasmic beta-catenin accumulation as a predictor of hematogenous metastasis in human colorectal cancer," Oncology, vol. 59, no. 4, pp. 302-309, 2000.

[17] S. Urakami, H. Shiina, H. Enokida et al., "Epigenetic inactivation of Wnt inhibitory factor-1 plays an important role 
in bladder cancer through aberrant canonical Wnt $/ \beta$-catenin signaling pathway," Clinical Cancer Research, vol. 12, no. 2, pp. 383-391, 2006.

[18] J. N. Kloth, G. J. Fleuren, J. Oosting et al., "Substantial changes in gene expression of Wnt, MAPK and TNF $\alpha$ pathways induced by TGF- $\beta 1$ in cervical cancer cell lines," Carcinogenesis, vol. 26, no. 9, pp. 1493-1502, 2005.

[19] J. R. Jass, K. G. Biden, M. C. Cummings et al., "Characterisation of a subtype of colorectal cancer combining features of the suppressor and mild mutator pathways," Journal of Clinical Pathology, vol. 52, no. 6, pp. 455-460, 1999.

[20] T. Kawasaki, K. Nosho, M. Ohnishi et al., "Correlation of $\beta$-catenin localization with cyclooxygenase-2 expression and CpG island methylator phenotype (CIMP) in colorectal cancer," Neoplasia, vol. 9, no. 7, pp. 569-577, 2007.

[21] G. Pelosi, A. Scarpa, G. Puppa et al., "Alteration of the Ecadherin/ $\beta$-catenin cell adhesion system is common in pulmonary neuroendocrine tumors and is an independent predictor of lymph node metastasis in atypical carcinoids," Cancer, vol. 103, no. 6, pp. 1154-1164, 2005.

[22] H. Taniguchi, H. Yamamoto, T. Hirata et al., "Frequent epigenetic inactivation of Wnt inhibitory factor-1 in human gastrointestinal cancers," Oncogene, vol. 24, no. 53, pp. 7946-7952, 2005.

[23] J. Paluszczak, J. Sarbak, M. Kostrzewska-Poczekaj et al., “The negative regulators of Wnt pathway-DACH1, DKK1, and WIF1 are methylated in oral and oropharyngeal cancer and WIF1 methylation predicts shorter survival," Tumor Biology, vol. 36, no. 4, pp. 2855-2861, 2015.

[24] K. Kawakami, H. Hirata, S. Yamamura et al., "Functional significance of Wnt inhibitory factor-1 gene in kidney cancer," Cancer Research, vol. 69, no. 22, pp. 8603-8610, 2009.

[25] W. L. Lambiv, I. Vassallo, M. Delorenzi et al., "The Wnt inhibitory factor 1 (WIF1) is targeted in glioblastoma and has a tumor suppressing function potentially by induction of senescence," Neuro-Oncology, vol. 13, no. 7, pp. 736-747, 2011.

[26] E. M. Rubin, Y. Guo, K. Tu, J. Xie, X. Zi, and B. H. Hoang, "Wnt inhibitory factor 1 decreases tumorigenesis and metastasis in osteosarcoma," Molecular Cancer Therapeutics, vol. 9, no. 3, pp. 731-741, 2010.

[27] J. Mazieres, B. He, L. You et al., "Wnt inhibitory factor-1 is silenced by promoter hypermethylation in human lung cancer," Cancer Research, vol. 64, no. 14, pp. 4717-4720, 2004.

[28] M. S. Elston, A. J. Gill, J. V. Conaglen et al., "Wnt pathway inhibitors are strongly down-regulated in pituitary tumors," Endocrinology, vol. 149, no. 3, pp. 1235-1242, 2008.

[29] J. Cui, W. Jiang, S. Wang, L. Wang, and K. Xie, "Role of Wnt/ $\beta$ catenin signaling in drug resistance of pancreatic cancer," Current Pharmaceutical Design, vol. 18, no. 17, pp. 2464-2471, 2012.

[30] S. C. C. Wong, S. F. E. Lo, K. C. Lee, J. W. P. Yam, J. K. C. Chan, and W. L. Wendy Hsiao, "Expression of frizzled-related protein and Wnt-signalling molecules in invasive human breast tumours," The Journal of Pathology, vol. 196, no. 2, pp. 145-153, 2002.

[31] K. Okino, H. Nagai, M. Hatta et al., "Up-regulation and overproduction of DVL-1, the human counterpart of the Drosophila dishevelled gene, in cervical squamous cell carcinoma," Oncology Reports, vol. 10, no. 5, pp. 1219-1223, 2003.

[32] V. Sherwood, "WNT signaling: an emerging mediator of cancer cell metabolism?” Molecular and Cellular Biology, vol. 35, no. 1, pp. 2-10, 2015.
[33] T. Fukui, M. Kondo, G. Ito et al., "Transcriptional silencing of secreted frizzled related protein 1 (SFRP1) by promoter hypermethylation in non-small-cell lung cancer," Oncogene, vol. 24, no. 41, pp. 6323-6327, 2005.

[34] P. N. Le, J. D. McDermott, and A. Jimeno, “Targeting the Wnt pathway in human cancers: therapeutic targeting with a focus on OMP-54F28," Pharmacology and Therapeutics, vol. 146, pp. 1-11, 2015.

[35] F. Pez, A. Lopez, M. Kim, J. R. Wands, C. C. de Fromentel, and P. Merle, "Wnt signaling and hepatocarcinogenesis: molecular targets for the development of innovative anticancer drugs," Journal of Hepatology, vol. 59, no. 5, pp. 1107-1117, 2013.

[36] I. Gehrke, R. K. Gandhirajan, and K.-A. Kreuzer, “Targeting the WNT/ $\beta$-catenin/TCF/LEF1 axis in solid and haematological cancers: multiplicity of therapeutic options," European Journal of Cancer, vol. 45, no. 16, pp. 2759-2767, 2009.

[37] G. H. Enders, "Wnt therapy for bone loss: golden goose or Trojan horse?" The Journal of Clinical Investigation, vol. 119, no. 4, pp. 758-760, 2009. 


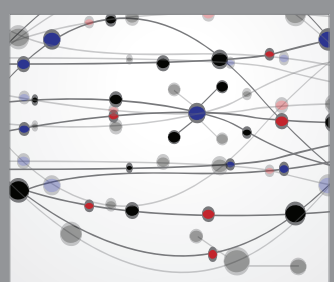

The Scientific World Journal
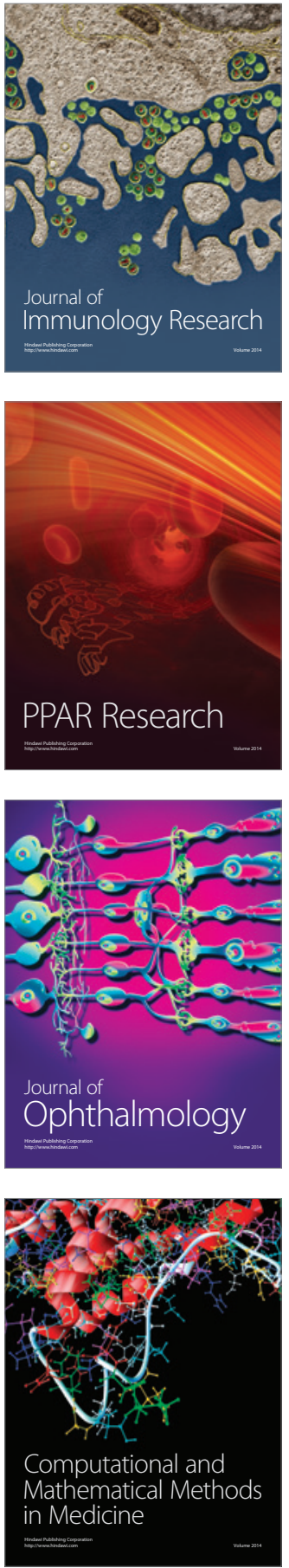

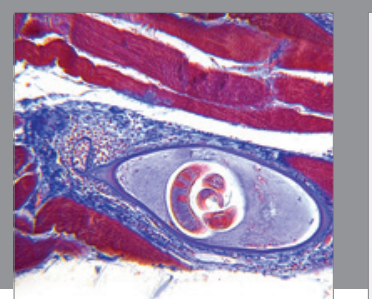

Gastroenterology Research and Practice

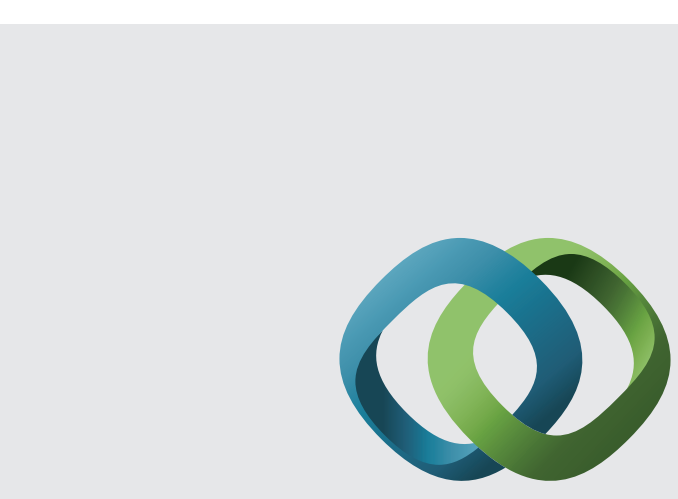

\section{Hindawi}

Submit your manuscripts at

http://www.hindawi.com
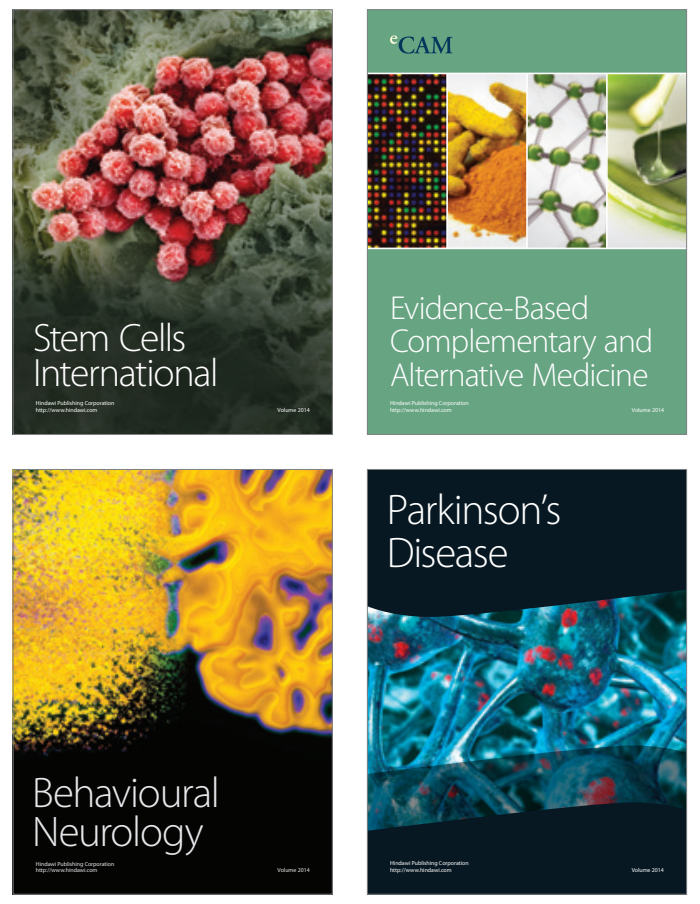
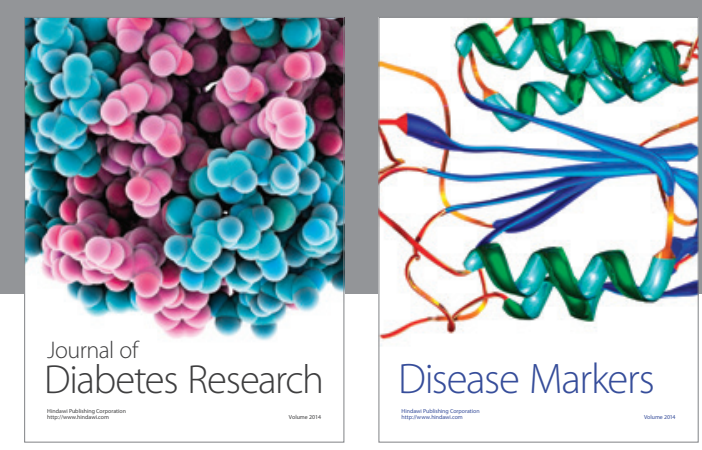

Disease Markers
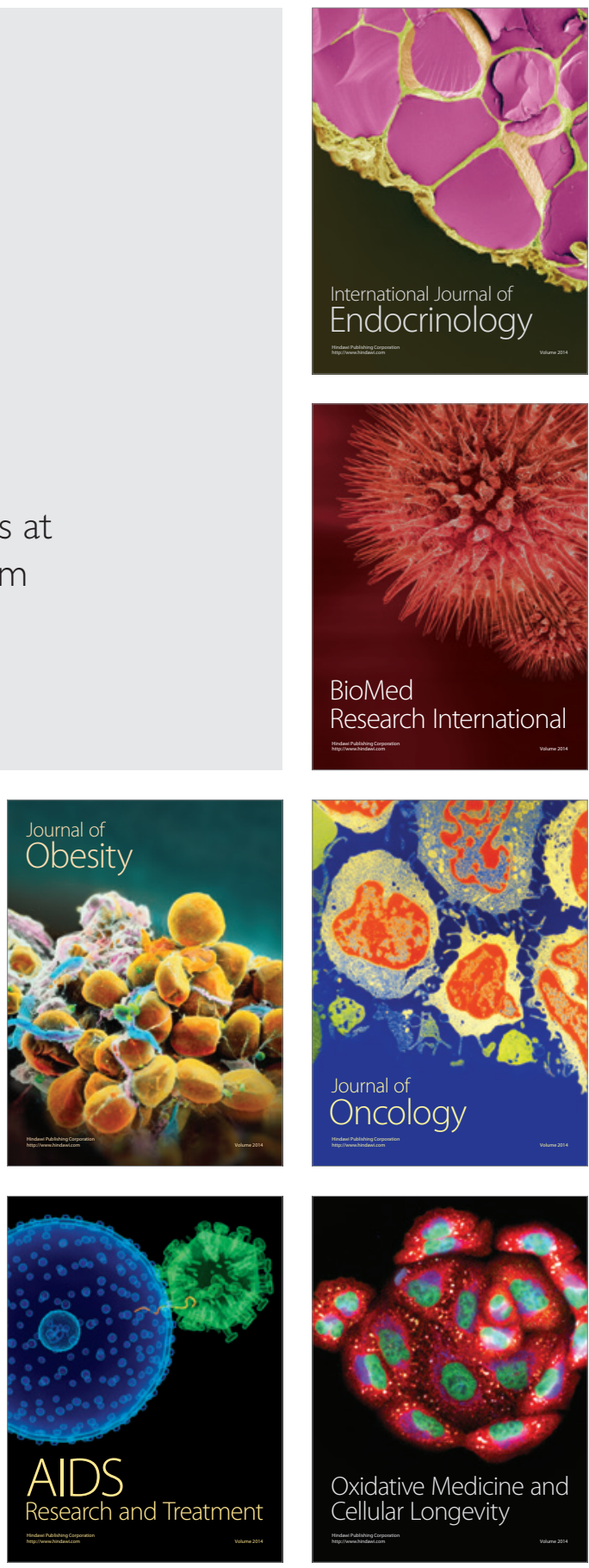